BULL. AUSTRAL. MATH. SOC.

VOL. $29(1984), 429-430$.

\title{
Mathematical MODELLing OF RATE-Limiting MECHANisms OF PYRITIC OXIDATION IN OVERBURDEN DUMPS
}

\author{
GREgORY BRUCE DAVIS
}

The mining of valuable minerals often leads to major environmental pollution problems especially when pyritic material is associated with the orebody being mined. In this thesis mathematical equations are formulated which describe a "dual region" model of pyritic oxidation within a waste rock dump, where it is assumed that oxygen transport is the rate-limiting step in the oxidation process, and that oxygen transport to reaction sites occurs in two stages; bulk diffusion of oxygen from the atmosphere, at the top surface of the dump, through the pore space of the dump to particles within the dump, and then diffusional transport of oxygen from the surface of particles to oxidation sites within the particles.

A moving boundary (shrinking-core) formulation, describing transport and reaction processes within a single particle of the dump, is solved by a new analytic series solution technique involving a change of coordinates leading to a non-linear partial differential equation with fixed boundaries. Based on this analysis a pseudo-steady state assumption is made with respect to diffusion within the particles giving a simplified system of equations for the dump model.

The system of equations is solved both in an approximate analytic way and numerically. An approximate analytic result is obtained by solving a planar moving boundary formulation which in turn comes from approximation

Received 16 January 1984 . Thesis submitted to University of Wollongong April 1983. Degree approved January 1984. Supervisors: Dr J. M. Hill and Dr A.I.M. Ritchie.

Copyright Clearance Centre, Inc. Serial-fee code: 0004-9727/84 $\$ A 2.00+0.00$. 
of the position of the moving reaction front within the particles of the dump. The position of the planar front within the dump is given by a transcendental equation which is shown to collapse on the (time) classical planar moving boundary result when the particle size tends to zero. A combined Crank-Nicolson and Backward Euler discretisation of the system of equations is adopted for the numerical scheme. The approximate and numerical solutions are compared.

A particle size distribution is included in the model equations. A substantial difference is observed, in parameter prediction, between a dump assumed to be comprised of particles of a single or "average" size, and a dump when a range of particle sizes is accounted for.

Variables such as the total sulphate production rate, the position of the planar moving front within the dump, the oxygen concentration within the pore space of the dump and the heat source distribution, are evaluated and their dependence on other parameters in the model noted. Comparisons are made with both experimental measurements and with predictions of a simpler diffusion rate-limited model of pyritic oxidation. The present model is in much better qualitative agreement with experimental values than the simpler model. The model is also extended to assess the effectiveness of a rehabilitation strategy intended to reduce pollutant production within oxidising pyritic material.

Division of Groundwater Research, CSIRO, Private Bag, PO, Wemb ley, Western Australia 6014 , Australia. 